\title{
Aortic dissection assessment by 4D phase-contrast MRI with hemodynamic parameters: the impact of stent type
}

\author{
Chien-Wei Chen ${ }^{1,2}$, Yuan-Hsi Tseng ${ }^{3,4}$, Chien-Chao Lin $^{3,4}$, Chih-Chen Kao ${ }^{3,4}$, Min Yi Wong ${ }^{3}$, Hua Ting ${ }^{1}$, \\ Yao-Kuang Huang ${ }^{3,4} \wedge$
}

${ }^{1}$ Institute of Medicine, Chung Shan Medical University, Taichung; ${ }^{2}$ Department of Diagnostic Radiology, Chang Gung Memorial Hospital Chiayi Branch, College of Medicine, Chang Gung University, Chiayi and Taoyuan; ${ }^{3}$ Division of Thoracic and Cardiovascular Surgery, Chia Yi Chang Gung Memorial Hospital, Chiayi; ${ }^{4}$ Chang Gung University, College of Medicine, Taoyuan

Correspondence to: Yao-Kuang Huang, MD, PhD. Division of Thoracic and Cardiovascular Surgery, Chia Yi Chang Gung Memorial Hospital, 6 West Section, Chia-Pu Rd, Putzu City, Chiayi County. Email: huang137@icloud.com.

Background: To explore the diagnostic performance of 4-dimensional phase-contrast magnetic resonance imaging (4D PC-MRI) in evaluating aortic dissection in different clinical scenarios.

Methods: The study group comprised 32 patients with a known aortic dissection who each underwent computed tomography angiography (CTA), and then 4D PC-MRI with a 1.5-T MR scanner. The 4D PCMRI images were compared with the CTA images to evaluate the aortic size, branch identification, and iliac and femoral arterial access.

Results: The patients were divided into three groups: (I) patients diagnosed with Type B aortic dissection but did not undergo intervention ( $\mathrm{n}=8$ ); (II) patients with residual aortic dissection after open repair of Type A dissection ( $\mathrm{n}=7$ ); (III) patients who underwent endovascular aortic repair with or without open surgery $(n=17)$. Without radiation or contrast media injection, 4D PC-MRI provided similar aortic images for patients in Group 1 and most of those in Group 2. In Group 3, stainless steel stents affected image quality in three patients. High-quality 4D PC-MRI images were obtained for the remaining 14 patients in Group 3, who had non-stainless steel stents, and provided major aortic information comparable to that provided by CTA with contrast media. The hemodynamic parameters of true and false lumens were evaluated between three patients with Type B aortic dissections and three patients who underwent thoracic endovascular aortic repair for their aortic dissection. The stroke volume was higher in the true lumen of the patients with stentgrafts than in the patients with Type B aortic dissection without intervention. The regurgitant fraction, an indicator of nonlaminar flow, was higher in the false lumens than in the true lumens. All 32 patients in this study tolerated 4D PC-MRI without adverse events.

Conclusions: 4D PC-MRI is radiation- and contrast media-free option for imaging aortic dissection. It not only provided images comparable in quality to those obtained with CTA but also provided information on hemodynamic parameters, including endoleak detection after thoracic endovascular aortic repair. 4D PCMRI was safe and accurate in evaluating chronic Type B aortic dissection and residual aortic dissection after surgery for acute Type A aortic dissection. Therefore, it could be a potential tool in treating pathology in aortic dissection, especially for patients with malperfusion syndrome of visceral vessels and in young patients with renal function impairment. However, certain endograft materials, especially stainless steel, may prevent the further application of 4D PC-MRI and should be avoided.

Keywords: 4-dimensional imaging; aortic dissection, endovascular repair; malperfusion; non-contrast imaging; magnetic resonance imaging (MRI)

^ ORCID: 0000-0003-2699-2207. 
Submitted May 17, 2020. Accepted for publication Sep 18, 2020.

doi: 10.21037/qims-20-670

View this article at: http://dx.doi.org/10.21037/qims-20-670

\section{Introduction}

Acute aortic syndrome is a critical public health concern with economic and social consequences. It includes several different conditions, such as intramural hematoma, perforated aortic ulcer, and the two types of aortic dissection, $\mathrm{A}$ and $\mathrm{B}$, of which Type $\mathrm{A}$ is considered to be the most severe (1-4). Computed tomography angiography (CTA) is a rapid imaging technique that is less invasive than conventional aortography and is the most widely chosen diagnostic method for patients with possible acute aortic pathology. However, CTA still requires the use of contrast media and radiation (5-8).

Contrast-enhanced magnetic resonance angiography (CE-MRA) is highly sensitive for detecting pathology in various blood vessels compared with conventional angiography. Magnetic resonance imaging (MRI) does not involve radiation exposure, but the contrast agents have undesirable effects. Nephrogenic systemic fibrosis (NSF) is a rare but severe complication of using gadoliniumbased contrast agents (GBCAs) in patients with pre-existing impairment of renal function $(9,10)$. Additionally, safety issues and imaging artifacts caused by prostheses are still a major concern $(11,12)$. Four-dimensional (three spatial dimensions combined with time) phase-contrast MRI (4D PC-MRI) is a non-contrast-enhanced MRI technique that can measure the velocity of blood flow in the aorta in any direction, and functional assessment of blood flow is possible by calculating the blood flow volume and flow pattern. This technique provides not only anatomical information but also hemodynamic status without the use of contrast agents. In this study, we summarize our preliminary experience of the clinical application of 4D PC-MRI in patients with aortic dissection, with a focus on the effect of the aortic stent graft after thoracic endovascular aortic repair (TEVAR) on image quality.

\section{Methods}

\section{Patients}

The Institutional Review Board of Chang Gung Memorial Hospital, a tertiary hospital, approved the study (IRB No. 201901776B0). All patients gave informed consent for the examinations. We prospectively collected information on consecutive patients evaluated using 4D PC-MRI for aortic pathology between April 2017 and February 2019. Patients were eligible for inclusion if they had a clinical indication for CTA of the aortic dissection. The exclusion criteria were non-MRI-compatible ferromagnetic devices and pregnancy. Also, patients with poor compliance or unable to lie down for the MRI protocol were excluded. Initially, 34 patients were evaluated: one patient was excluded due to fever at the scheduled time for imaging, and another was unable to lie down due to complicated spinal disease. All patients underwent CTA scanning with a 64-slice multidetector scanner (Somatom Sensation 64; Siemens Healthcare, Erlangen, Germany) with intravenous administration of contrast media, followed by 4D PC-MRI to assess aortic pathology.

\section{MRI protocol}

With the patient in a supine position, conventional MRI was performed with a 1.5-Tesla scanner (Ingenia Rev R5 V30-rev.02; Philips, Amsterdam, The Netherlands), and an electrocardiogram (ECG) gating system. First, we obtained anatomical scans of the blood vessels and aortic dissection area with three planes scanned separately, using T2 turbo spin echo scanning with the following parameters: singleshot mode; repetition time repetition (TR), shortest; echo time (TE), shortest; voxel size, $0.6 \times 0.84 \times 4 \mathrm{~mm}^{3}$; number of signals averaged (NSA), 1; scan duration, $1 \mathrm{~min}$. We also used balanced turbo field echo scanning with the following parameters: single-shot mode; TR, shortest; TE, shortest; voxel size, $1.84 \times 1.87 \times 8 \mathrm{~mm}^{3}$; NSA, 1 ; scan duration, $1 \mathrm{~min}$. The axial range included the aortic arch to the aortic bifurcation level, the coronal field comprised the entire heart and aorta, and the oblique sagittal field contained the whole aorta and parallel aortic arch. The two-dimensional images helped to understand the type and extent of the aortic dissection and were the basis for subsequent 4D PCMRI scanning with the following parameters: $3 \mathrm{D}$ turbo field echo (TFE); TR, shortest; TE, shortest; flip angle, $5^{\circ}$; voxel size, $2.25 \times 2.25 \times 3 \mathrm{~mm}^{3}$; and PC velocity, $120 \mathrm{~cm} / \mathrm{s}$; scan duration, $6.02 \mathrm{~min}$. The imaging section had to include the aortic arch and descending aorta. The 4D images helped to 

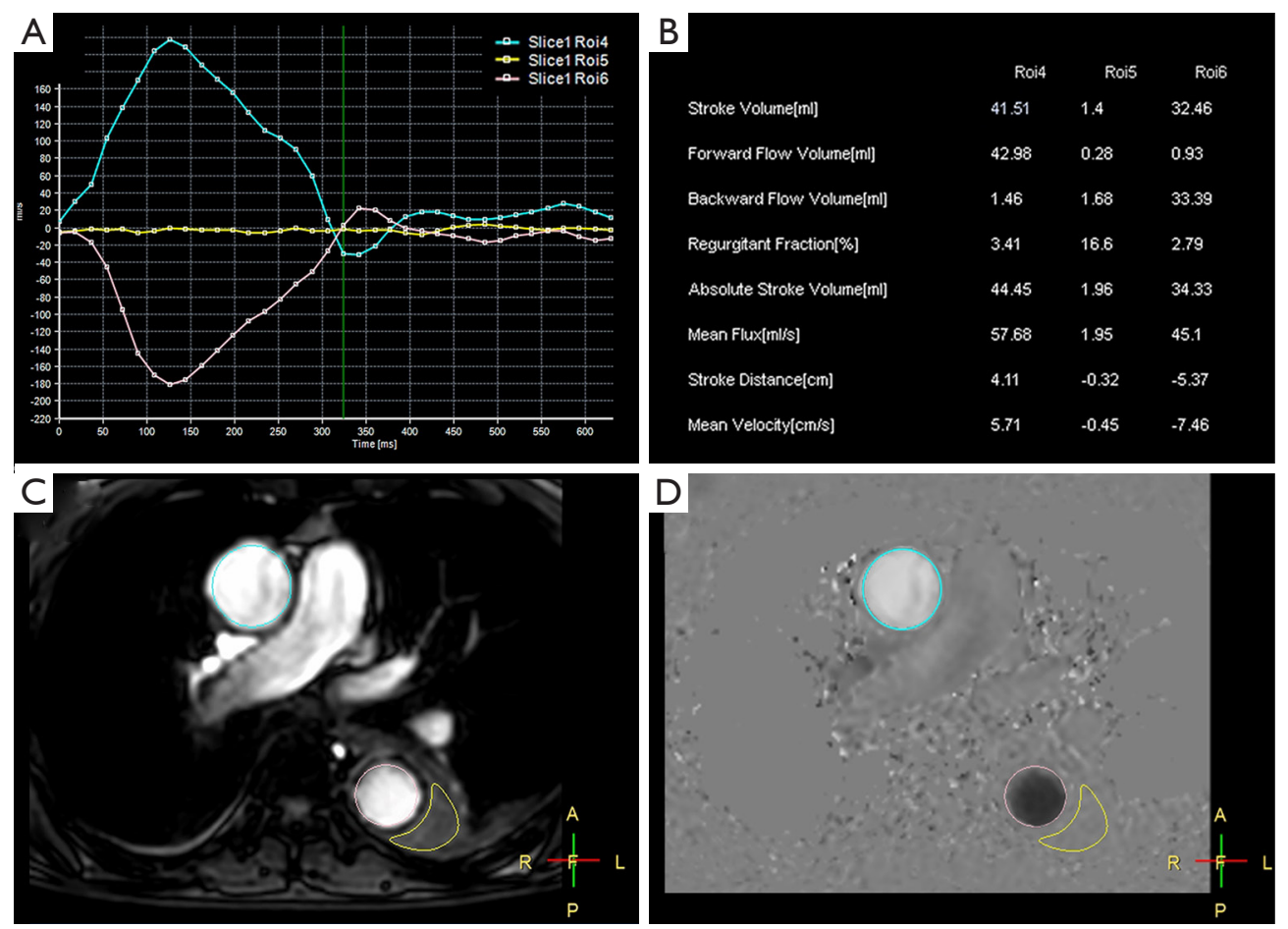

Figure 1 Phase-contrast MRI (PC-MRI) with hemodynamic analysis. (A) Flow velocities as a function of time in the ascending aorta and descending aorta of a participant using electrocardiogram-gated PC-MRI; (B) hemodynamic parameters, including stroke volume, forward flow volume, backward flow volume, regurgitant fraction, absolute stoke volume, mean flux, stroke distance, stroke distance, and mean velocity; (C) drawing the region of interest (ROI) on the ascending aorta and the false and true lumens of the descending aorta (covering the whole lumen); (D) velocity-encoded image, with flows from inferior to superior depicted as white and flows from superior to inferior as black.

understand the spatial anatomy of the artery. A quantitative flow (Q-flow) scan on a plane perpendicular to blood flow was then performed with the following parameters: scan technique, TFE PC; TR, shortest; TE, shortest; flip angle, $12^{\circ}$; slice thickness, $8 \mathrm{~mm}$; the field of view, $248 \times 300$; and PC velocity, $200 \mathrm{~cm} / \mathrm{s}$; scan duration, $13 \mathrm{~s}$ with breath hold. All 4D PC-MRI images were acquired without the use of GBCA. Quantitative analysis was performed by drawing the region of interest (ROI) on the false and true lumens (covering the whole lumen) at the level of the ascending aorta, aortic arch, descending aorta, and abdominal aorta. Q-flow variables included stroke volume, forward flow volume, backward flow volume, regurgitant fraction, absolute stoke volume, mean flux, stroke distance, stroke distance, and mean velocity (Figure 1).

\section{Results}

Between April 2017 and March 2020, 32 patients were enrolled and evaluated using 4D PC-MRI for aortic pathology [ 28 male, 4 female; age range $30-79$ years (mean age, $56.2 \pm 12.8$ years)]. Details of age, sex, comorbidities, and aortic condition are listed in Table 1 . All 32 patients tolerated 4D PC-MRI without adverse events, despite almost all of them having hypertension and 9 having renal diseases (chronic renal insufficiency, renal infarction with atrophy, polycystic kidney disease, and autoimmune nephritis). The patients were further classified into three clinical scenarios: Type B aortic dissection without intervention ( $\mathrm{n}=8$, Group 1); residual aortic dissection after open repair of Type A aortic dissection ( $\mathrm{n}=7$, Group 2); and aortic dissecting aneurysm with TEVAR ( $\mathrm{n}=17$, Group 3). The interval between the onset of aortic dissection to 4D PC-MRI ranged from 1 to 125 months.

We use the Kolmogorov-Smirnov test for testing the normality of the measured values and then used the paired $t$-test for comparing the means. The measured values of maximal diameter on CTA and 4D PC-MRI showed 
Table 1 Demographic data of 32 patients evaluated by 4D-PC MRI

\begin{tabular}{lc}
\hline Variable & Value \\
\hline Age (years old) & $56.2 \pm 12.8$ \\
Sex (male) & 28 \\
Comorbidities (number) & 27 \\
Hypertension & 12 \\
Renal disease & 1 \\
DM & 2 \\
COPD & 1 \\
Marfan syndrome & 2 \\
Stroke and spinal ischemia & 0 \\
Coronary artery disease & \\
Aortic intervention & 8 \\
Type B aortic dissections without intervention & 7 \\
Open surgery for Type A aortic dissections & 17 \\
without aortic stent & \\
With endovascular aortic repair & diabetes \\
\hline COPD, chronic obstructive pulmonary disease; DM, \\
mellitus.
\end{tabular}

a normal distribution. The measured maximum aortic diameter by 4D-PC MRI [mean \pm standard deviation (SD), $51.34 \pm 7.61 \mathrm{~mm}]$ was significantly higher than the measured maximum aortic diameter by $4 \mathrm{D}-\mathrm{PC}$ MRI (mean $\pm \mathrm{SD}$, $50.62 \pm 7.54 \mathrm{~mm})(\mathrm{P}=0.013)$. The effectiveness of 4D PCMRI versus CTA with contrast media was compared in the three groups. All patients in Group 1 (nonintervention) had chronic aortic dissections, the duration of aortic dissection ranged from 23 to 125 months, and the difference in maximum aortic diameter measured between 4D-PC MRI and CTA was $0.53 \pm 0.99 \mathrm{~mm}$ (mean $\pm \mathrm{SD}$ ) (Table 2). Notably, three patients in Group 1 (3/8, 37.5\%) had renal insufficiency. In the patients who underwent open surgery for Type A aortic dissections without endovascular aortic repair (Group 2), the difference in maximum aortic diameter measured between 4D-PC MRI and CTA was $0.59 \pm 2.04 \mathrm{~mm}$ (Table 3). Seventeen patients underwent 4D-PC MRI after TEVAR and were assigned to Group 3 (Table 4). The image quality of 4D PC-MRI was inadequate in three patients (cases 3, 6, and 9), and only the iliac arterial status could be identified in these images (Figure 2). The endovascular device used in three patients was the Cook

Table 2 Aortic dissection without surgical or endovascular intervention

\begin{tabular}{|c|c|c|c|c|c|c|}
\hline Patient No. & Sex/age & Initial diagnosis & Comorbidities & $\begin{array}{l}\text { Period from diagnosis to } \\
\text { 4D PC-MRI (month) }\end{array}$ & $\begin{array}{l}\text { Maximal diameter } \\
\text { on CTA }(\mathrm{mm})\end{array}$ & $\begin{array}{l}\text { Maximal diameter on } \\
\text { 4D PC-MRI (mm) }\end{array}$ \\
\hline 1 & $\mathrm{M} / 59$ & $\begin{array}{l}\text { Type B aortic } \\
\text { dissection }\end{array}$ & $\begin{array}{l}\text { CRF (left renal } \\
\text { infarction), HTN }\end{array}$ & 78 & 45 & 46.3 \\
\hline 2 & $M / 59$ & $\begin{array}{l}\text { Type B aortic } \\
\text { dissection }\end{array}$ & HTN & 125 & 43 & 45 \\
\hline 4 & $M / 56$ & $\begin{array}{l}\text { Type B aortic } \\
\text { dissection }\end{array}$ & $\begin{array}{l}\text { Left lung mass by } \\
\text { tuberculosis, HTN }\end{array}$ & 31 & 43 & 42 \\
\hline 5 & $\mathrm{M} / 66$ & $\begin{array}{l}\text { Type B aortic } \\
\text { dissection }\end{array}$ & HTN & 23 & 53 & 54.2 \\
\hline 7 & $F / 42$ & $\begin{array}{l}\text { Type B aortic } \\
\text { dissection }\end{array}$ & HTN & 46 & 36 & 36.2 \\
\hline 8 & $M / 50$ & $\begin{array}{l}\text { Type B aortic } \\
\text { dissection }\end{array}$ & $\begin{array}{l}\text { CRF, HTN, FF } \\
\text { bypass for ischemic } \\
\text { leg }\end{array}$ & 95 & 65 & 65.3 \\
\hline
\end{tabular}

${ }^{*}$, major information including maximal dissecting aneurysmal diameter, false lumen location, visceral branches distributions and iliac artery dissections. CRF, chronic renal insufficiency; HTN, hypertension. 
Table 3 Patients with Type A aortic dissection after open surgical repair: evaluation by 4D phase-contrast MRI and CTA with contrast media

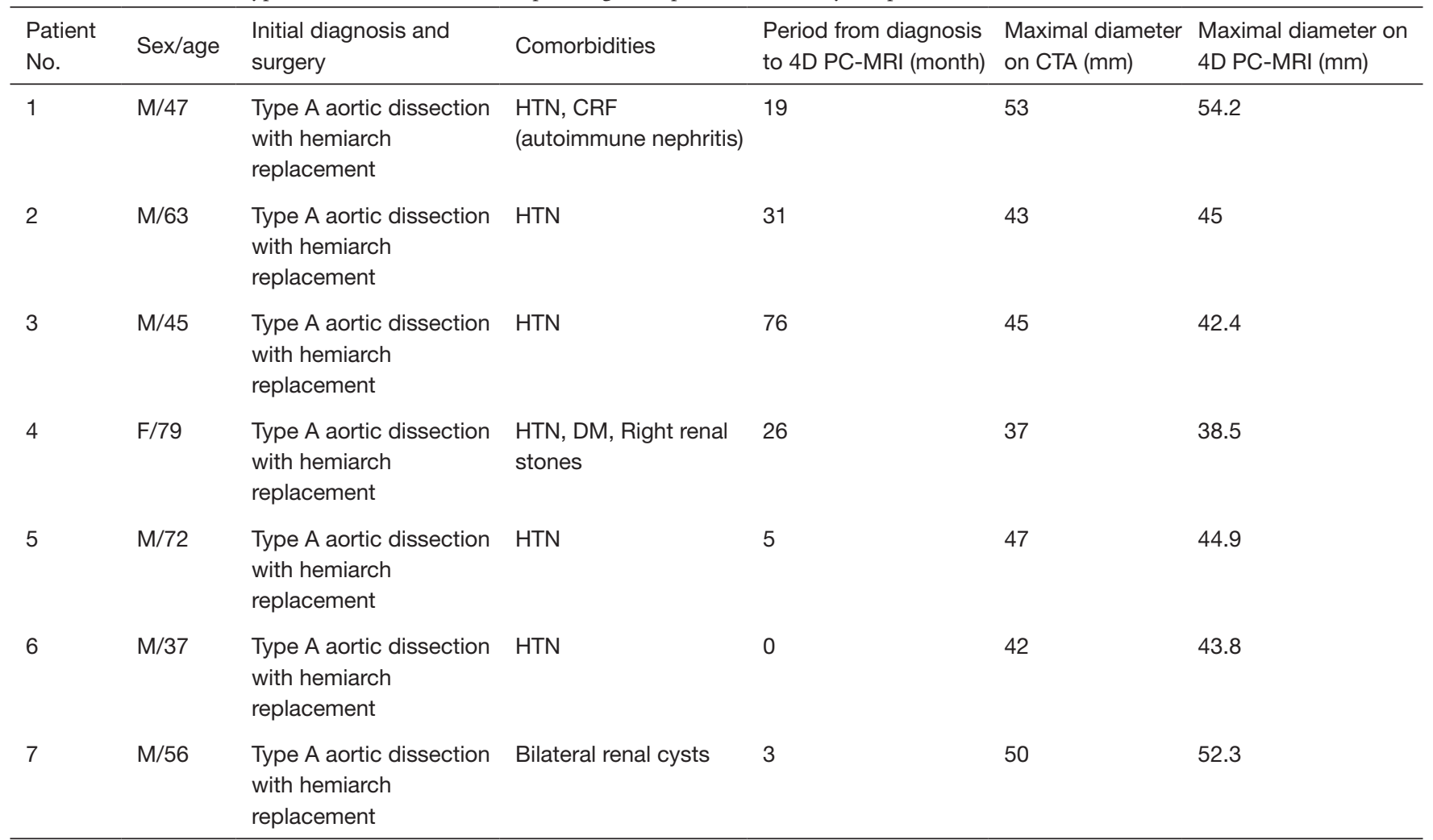

CRF, chronic renal insufficiency; DM, diabetes mellitus; HTN, hypertension.

Zenith ${ }^{\circledR}$ TX $2^{\circledR}$, and two patients also had mechanical aortic valves from previous Bentall procedures. The other 14 patients in Group 3 had similar imaging quality in 4D PCMRI that provided primary aortic information comparable to that provided by CTA with contrast media (Figure 3). Also, the difference in maximum aortic diameter measured between the two imaging modalities was $0.89 \pm 1.45 \mathrm{~mm}$. The 14 patients had Gore ${ }^{\circledR}$ TAG $^{\circledR}$ (TAG, TGT, and TGU) and Cook Zenith ${ }^{\circledR}$ dissection endovascular stents. Blandaltman plots showed that most of the measured differences were within the agreement (from -2.15 to 3.59 ), suggesting good agreement between 4D PC MRI and CTA (Figure 4). The interrater reliability of Cohen's kappa coefficient for examining the ability to detect aortic dissection between 4D PC MRI and CTA was 0.784 , indicating substantial agreement between the two imaging modalities.

The quantitative hemodynamic analysis was performed in 8 patients with type B aortic dissections and 14 patients with TEVAR. The results showed that the postTEVAR true lumen had higher absolute stroke volume (Figure $5 A$ ). As well, the regurgitant fraction, which indicates a nonlaminar flow pattern, was higher in the false lumens and lower in the true lumens in both type B aortic dissection and post-TEVAR patients (Figure 5B). Those findings are shown in a 4D flow visualization (Video 1).

\section{Discussion}

Conventional CT with contrast media has been regarded as the gold standard for the detection of aortic dissection. Modern CT scanners can provide submillimeter isotropic three-dimensional (3D) datasets within a single breathhold during the first pass of intravenous iodinated contrast $(13,14)$. Obtaining satisfactory arterial enhancement is crucial in the assessment of aortic dissections. Optimal IV contrast medium administration, bolus tracking technique (acquiring a precontrast image at a reference level with the placement of an ROI over a target vessel), dual-energy CT, ECG gating to reduce cardiac motion artifacts, and other modified techniques have further improved the quality of CTA aortic images $(15,16)$, and assist not only surgical planning for endovascular aortic repair but also 


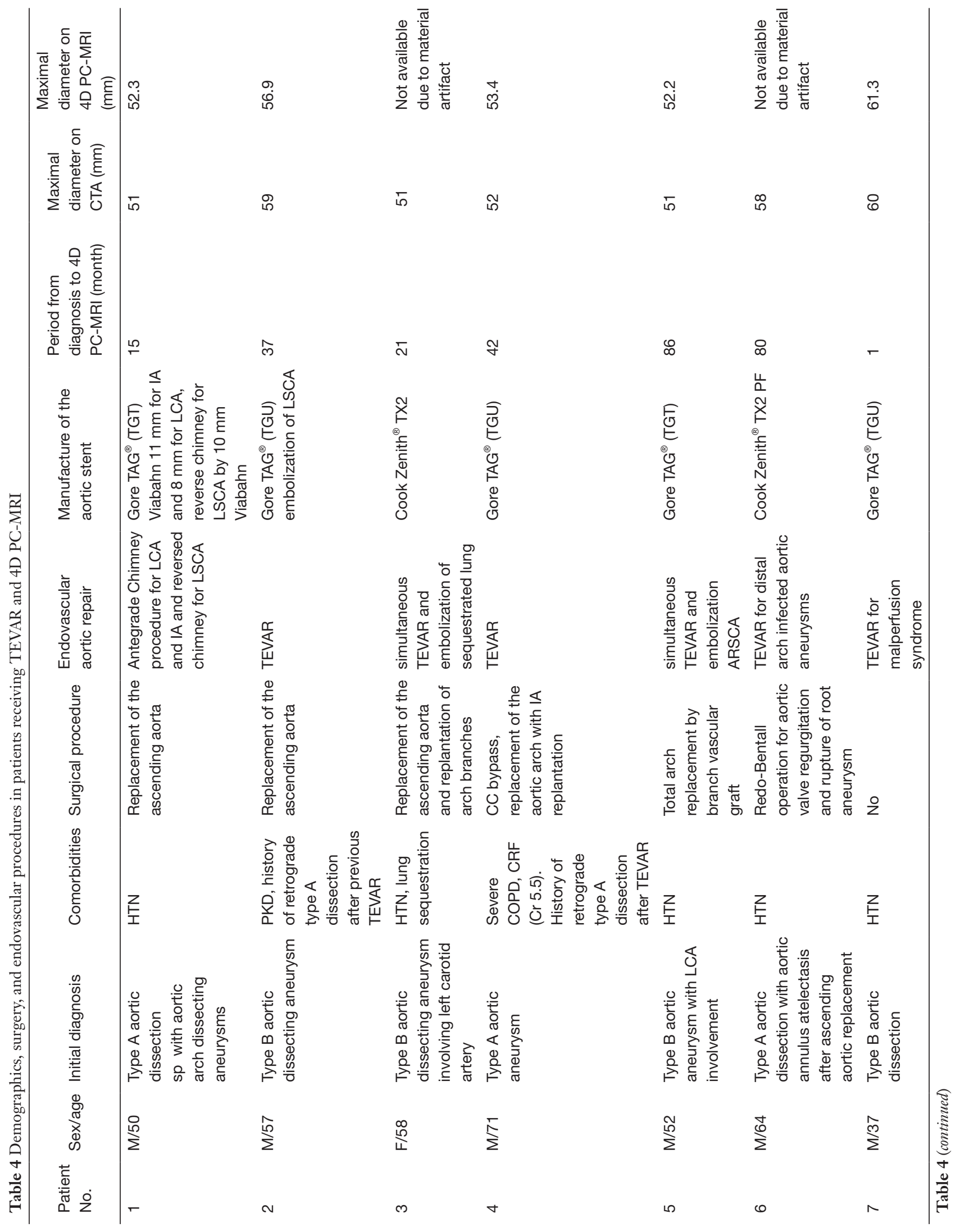




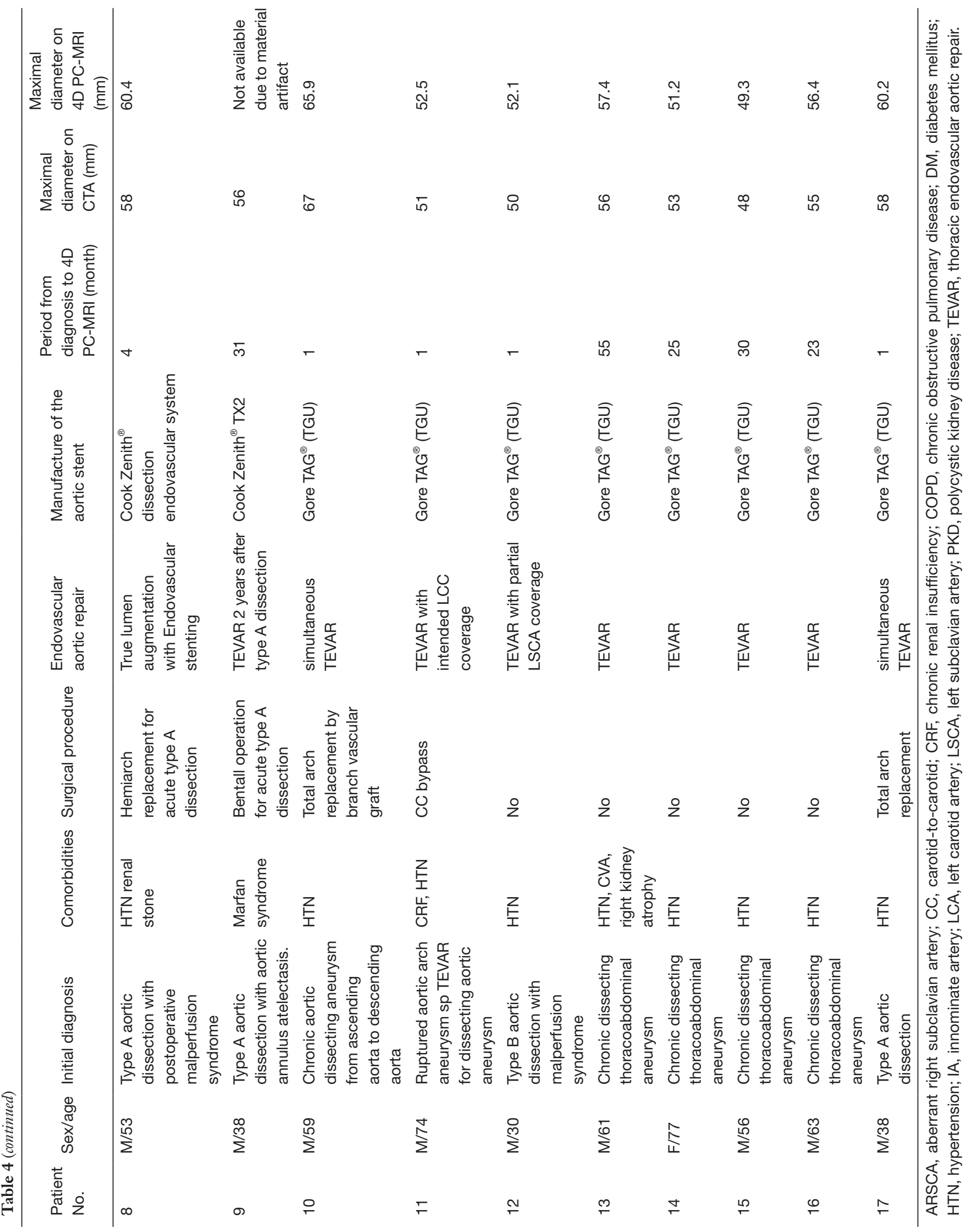



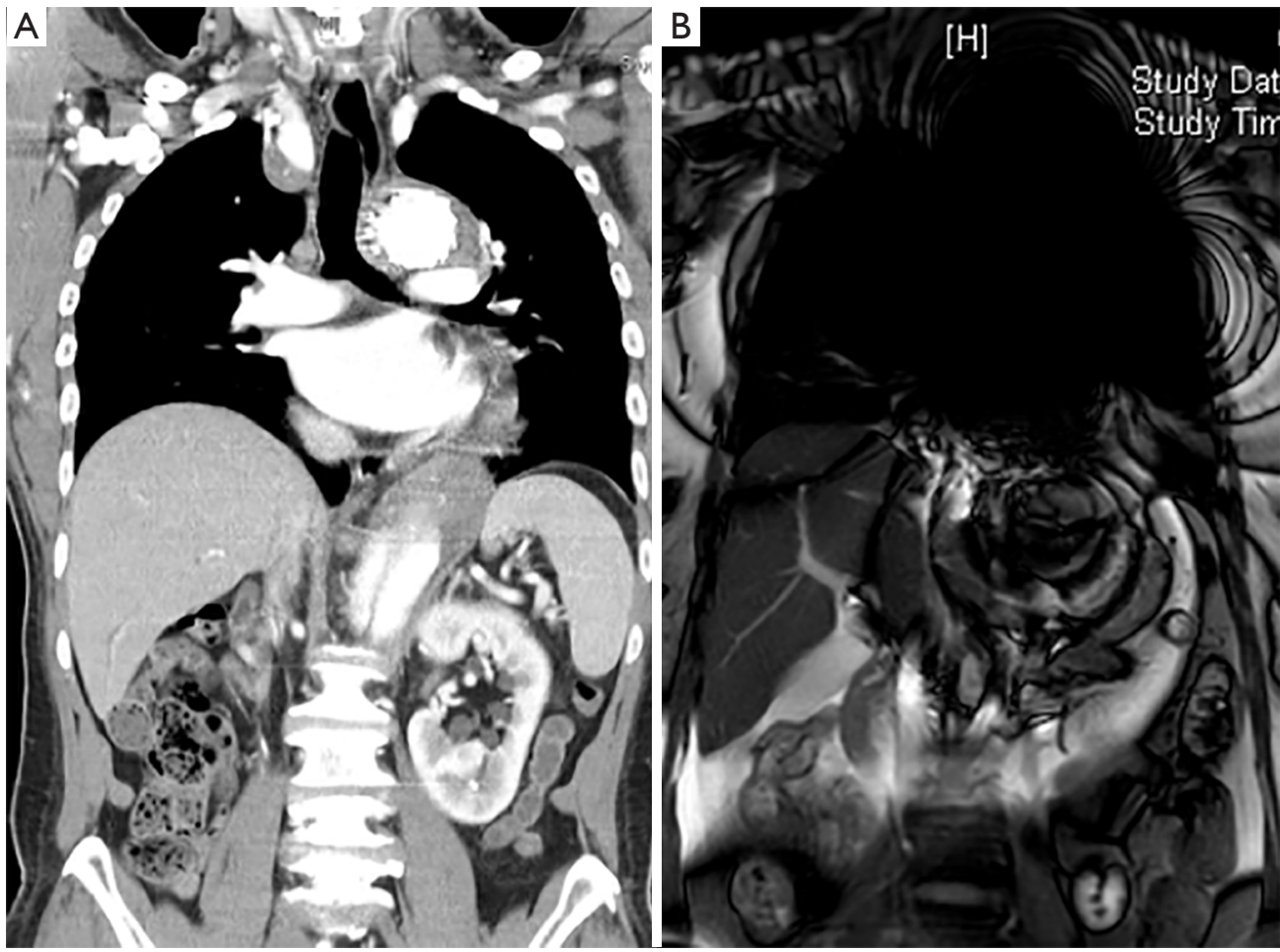

Figure 2 A 58-year-old woman with Type B aortic dissection and left lung sequestration underwent TEVAR with a Cook Zenith® CX2. (A) Computed tomography (CT) with injection of contrast media; (B) 4D phase-contrast MRI for aortic dissection showing significant artifact in the thoracic portion. CT, computed tomography; MRI, magnetic resonance imaging.
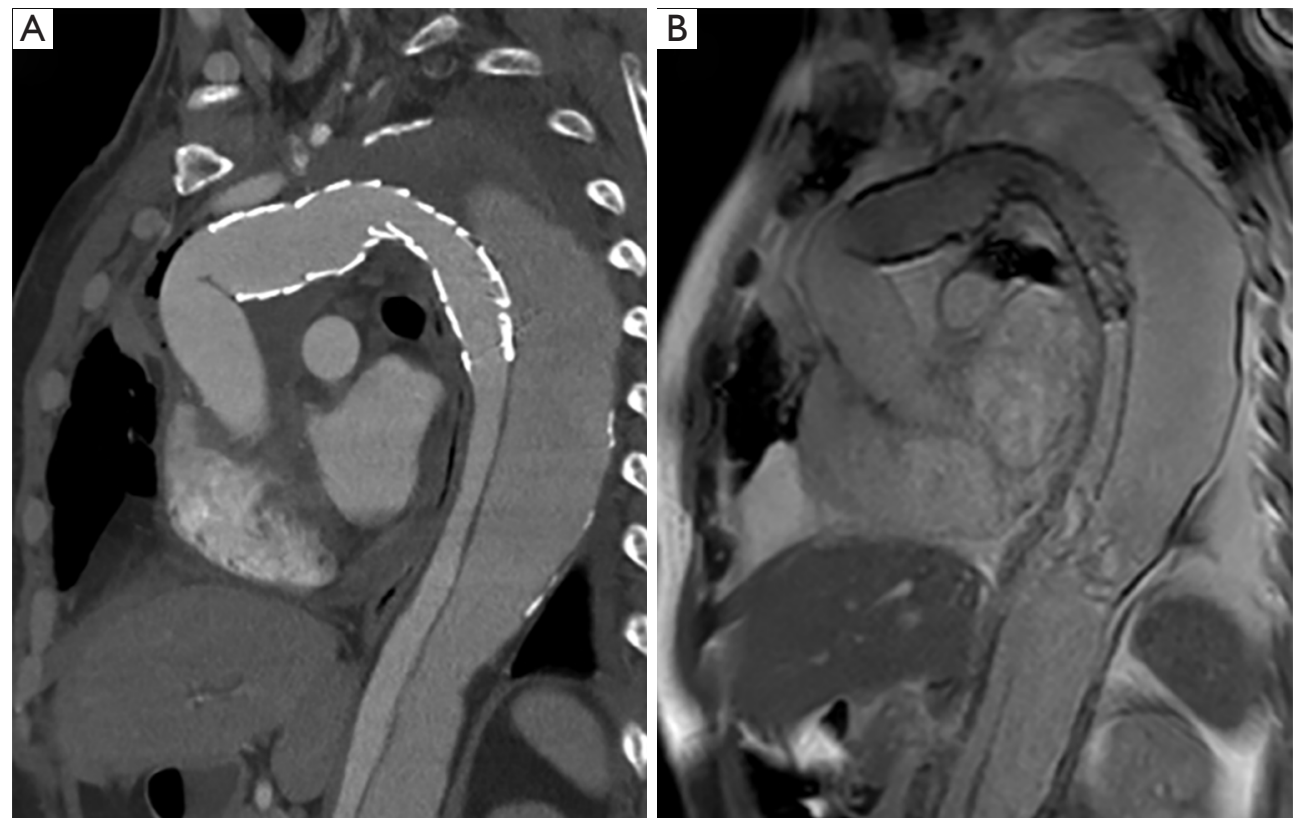

Figure 3 A 59-year-old man underwent total arch replacement for Type A aortic dissection and simulations TEVAR by Gore® TUG. (A) Contrast-enhanced CT showing the sagittal view of the aorta after stent placement; (B) MRI showing images similar to those with contrastenhanced CT, which can identify the false lumen and true lumen in the post-stenting aorta without ferromagnetic artifacts. CT, computed tomography; TEVAR, thoracic endovascular aortic repair. 
postoperative evaluation. However, CTA requires contrast media, an agent that can cause acute kidney injury. When patients have impaired renal function or unstable renal flow due to malperfusion syndrome, contrast media can only be given after mitigating the possible risk of acute renal failure on a case-by-case basis following risk-benefit analysis (17). Moreover, radiation dose accumulation is still a concern, especially in young patients (18-21).

However, unlike ultrasonography (US), CT does not provide information on blood flow direction and rate. Color Doppler US identifies the flow direction and is commonly used to detect aneurysms in emergency departments rapidly. Moreover, the US reveals real-time dynamic changes in endoleaks or aneurysms and is both low and radiationfree. However, the data quality of the US varies because of differences in operator skills and patient status. In particular, in patients with intestinal gas, obese, and thoracic aortic diseases, the US may not reliably diagnose aortic disease.

CE-MRA is highly sensitive for detecting pathology in various blood vessels but requires administration of

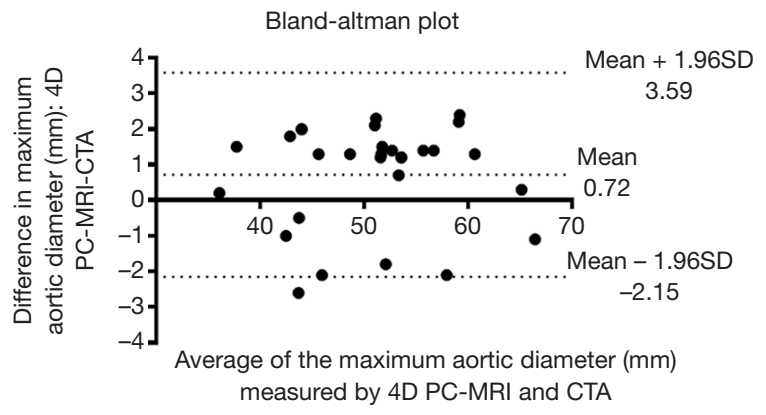

Figure 4 Bland-Altman plot showing most of the measured differences within the limits of agreement (LOA, from -2.15 to 3.59). a GBCA, which shortens longitudinal blood relaxation (T1). A rapid 3D T1-weighted spoiled gradient echo pulse sequence with a short repetition time and echo time is ideal for CE-MRA, providing images with a high signal-to-noise ratio and spatial resolution that are free from flow-related artifacts. Two acquisition modes are used in CE-MRA: single-phase and time-resolved (22). Single-phase MRA captures vascular images at a single point in time, whereas time-resolved MRA consists of multiple acquisitions of an imaged volume over successive time points post-GBCA administration. In time-of-fly MRA (TOF MRA), blood flow is used as the intrinsic contrast agent, and the signal is based on an in-flow effect. The signal in the vessel depends on the flow up to a threshold speed, which is calculated by the slice thickness, in millimeters, divided by repetition time, in milliseconds. However, the vessels can be observed most clearly when they are orthogonal to the $2 \mathrm{D}$ plane because in-plane vessels sometimes experience loss of signal $(20,21)$.

These types of CE-MRA do not involve radiation exposure, but the non-iodinated contrast agents (e.g., GBCA) still have undesirable effects. NSF is a rare but dangerous complication of GBCAs in patients with preexisting renal function impairment $(9,10,23)$. The recent discovery of cerebral gadolinium deposition in patients with normal renal function is also of concern, although the clinical significance of this phenomenon is yet to be determined (24).

4D PC-MRI is a newly developed technique that can, in a single scan, acquire flow information of the entire aortic volume over time (25). Blood flow can be visualized and quantified as it travels from the heart through the true and false lumens and entry tears of a dissection. It is radiation-
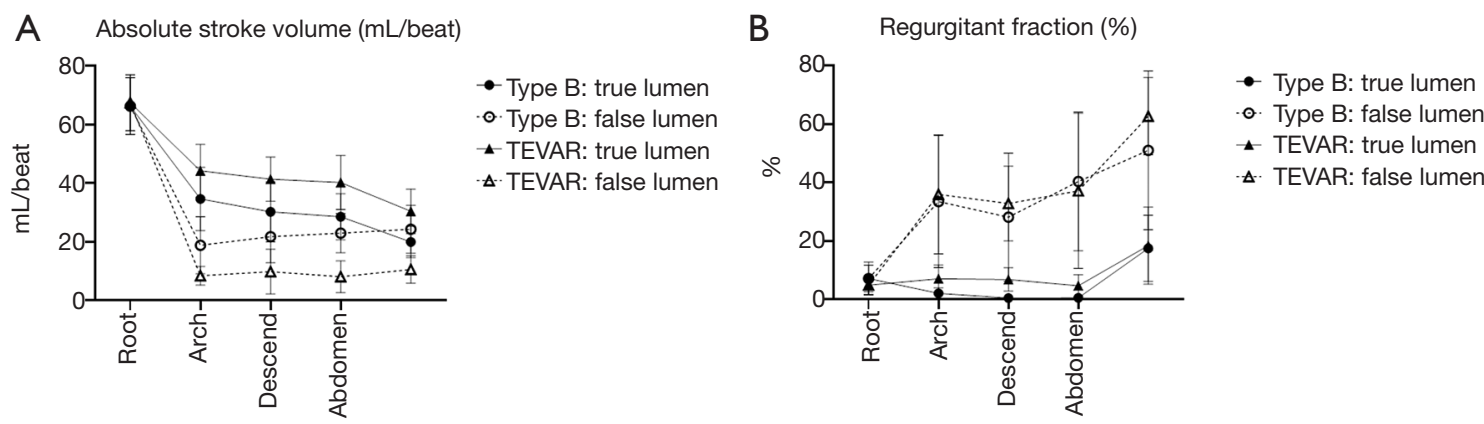

Figure 5 Preliminary analysis between patients with aortic stent graft and patients with dissection without intervention. (A) TEVAR patients show higher stroke volume in the true lumen than the patients without aortic intervention; (B) their false lumens shown higher regurgitant fraction than true lumens. TEVAR, thoracic endovascular aortic repair. 
free and does not require contrast media. Moreover, 4D PC-MRI can detail the relationship between the false lumen, true lumen, major visceral branches, and major communicators between the true and false lumens. Previous pioneering laboratory research demonstrated that 4D PCMRI at 3.0 T could evaluate aortic dissection, focusing on the hemodynamic parameters $(26,27)$. In this study, we further described its versatile application in clinical scenarios, which may help vascular surgeons to choose the right therapeutic plan using 4D PC-MRI at 1.5T. For patients with stable, chronic Type $\mathrm{B}$ aortic dissections, 4D PC-MRI provided similar aortic information as CTA without the need for radiation or contrast media injection (Table 2). Moreover, 4D PC-MRI could provide similar information as CTA after open surgery for type A aortic dissections (Table 3). It is an even more reasonable imaging option for young patients and those with poor renal function.

Endovascular aortic repair is the standard treatment of aortic dissecting pathology. However, carefully screening for this treatment is critical for improved outcomes. As the major postoperative complication, endoleaks are classified into five types based on the direction, location, and source of blood flow, and each type requires different treatment strategies (28). The advantage of detecting endoleaks after TEVAR by using 4D PC-MRI has been documented in our previous publication (29). In the present study, we found that the composition of aortic stents and coexisting prostheses (e.g., mechanical valves) affects image quality with 4D PC-MRI. In the three cases of poor-quality 4D PC MRI scans, the patient's endoprosthesis was made of stainless steel (Cook Zenith TX II). Image artifacts prevented the evaluation of the thoracic and abdominal aorta, with lesser effects on the iliac vessels. By contrast, artifacts were minimal in patients with nitinol-based endograft (Gore ${ }^{\circledR}$ TAG $^{\circledR}$ and Cook Zenith ${ }^{\circledR}$ dissection stent) (Video 1). Therefore, the Cook Zenith Alpha, a nitinol stent graft, has replaced the stainless-steel Cook Zenith ${ }^{\circledR}$ $\mathrm{TX} 2^{\circledR}$ in our institution. Although all instruction manuals of aortic endograft, even those made of stainless steel, assert that they are safe for MRI examinations, stainless steel aortic endografts should be avoided if 4D PC-MRI is being considered for use as a follow-up modality. In our preliminary hemodynamic analysis for 4D PC MRI, the stroke volume was higher in the true lumen of the patients with stent-grafts than in the patients with type B aortic dissection without intervention. And the regurgitant fraction, an indicator of nonlaminar flow, was higher in the false lumen than in the true lumen of all patients. These hemodynamic parameters need further investigation regarding their clinical application (Figure 4).

However, there are several disadvantages to the clinical application of 4D PC-MRI. First, the 4D PC-MRI data are acquired over a large volume and are susceptible to error from magnetic field inhomogeneities, Maxwell terms, eddy currents, and gradient nonlinearities. Second, motion artifacts from cardiac and respiratory motion are another potential source of error in 4D MRI acquisition. Finally, 4D MRI is relatively time consuming (processing time: $30 \mathrm{~min}$ ) and expensive, which prevents its application in acute aortic dissections.

\section{Study limitations}

We attempted to identify the clinical value and disadvantages of the application of 4D PC-MRI to aortic pathology. First, the major limitation is the nonrandomized study with few patients. Further studies with larger sample size and randomized design should be conducted. Second, although PC-MRI using quantitative analysis methods can provide various parameters to assess hemodynamic status, quantitative analysis probably includes useful information for determining the optimal therapeutic strategy for complex aortic diseases. However, this study lacked a computed streamline analysis for further assessment of the blood flow model in each participant. Further studies of quantitative analysis and streamline computation are needed, especially for evaluating the endoleak model, to assess the utility of full clinical application of 4D PC-MRI.

\section{Conclusions}

4D PC-MRI is radiation- and contrast media-free option for imaging aortic dissection. It provides not only images that were comparable in quality to those of CTA but also provided information on hemodynamic parameters, including endoleak detection after TEVAR. It is safe and accurate for chronic Type B aortic dissection and residual aortic dissection after acute Type A aortic dissection. Thus, 4D PC-MRI could be a powerful tool in treating aortic dissection, especially in malperfusion syndrome of visceral vessels and in young patients and those with impaired renal function. However, certain endograft materials, especially stainless steel, may prevent the further application of $4 \mathrm{D}$ 
PC-MRI and should be avoided.

\section{Acknowledgments}

This manuscript was edited by Wallace Academic Editing and QIMS English editing services.

Funding: This study was supported by Chang-Gung Memorial Hospital (Contract Nos. CMRPG6E0422, CORPG6G0091, CORPG6G0092, CORPG6D0292, and NMRPG6D6022).

\section{Footnote}

Conflicts of Interest: All authors have completed the ICMJE uniform disclosure form (available at http://dx.doi. org/10.21037/qims-20-670). The authors have no conflicts of interest to declare.

Ethical Statement: The Institutional Review Board of Chang Gung Memorial Hospital, a tertiary hospital, approved the study (IRB No. 201901776B0). All patients gave informed consent for the examinations.

Open Access Statement: This is an Open Access article distributed in accordance with the Creative Commons Attribution-NonCommercial-NoDerivs 4.0 International License (CC BY-NC-ND 4.0), which permits the noncommercial replication and distribution of the article with the strict proviso that no changes or edits are made and the original work is properly cited (including links to both the formal publication through the relevant DOI and the license). See: https://creativecommons.org/licenses/by-nc-nd/4.0/.

\section{References}

1. Lansman SL, Saunders PC, Malekan R, Spielvogel D. Acute aortic syndrome. J Thorac Cardiovasc Surg 2010;140:S92-7; discussion S142-S146.

2. Eggebrecht H, Plicht B, Kahlert P, Erbel R. Intramural hematoma and penetrating ulcers: indications to endovascular treatment. Eur J Vasc Endovasc Surg 2009;38:659-65.

3. Carpenter SW, Kodolitsch YV, Debus ES, Wipper S, Tsilimparis N, Larena-Avellaneda A, Diener H, Kolbel T. Acute aortic syndromes: definition, prognosis and treatment options. J Cardiovasc Surg (Torino) 2014;55:133-44.

4. Bossone E, LaBounty TM, Eagle KA. Acute aortic syndromes: diagnosis and management, an update. Eur Heart J 2018;39:739-749d.

5. Cerna M, Kocher M, Thomas RP. Acute aorta, overview of acute CT findings and endovascular treatment options. Biomed Pap Med Fac Univ Palacky Olomouc Czech Repub 2017;161:14-23.

6. Valente T, Rossi G, Lassandro F, Rea G, Marino M, Muto M, Molino A, Scaglione M. MDCT evaluation of acute aortic syndrome (AAS). Br J Radiol 2016;89:20150825.

7. Di Eusanio M, Russo V, Buttazzi K, Lovato L, Di Bartolomeo R, Fattori R. Endovascular approach for acute aortic syndrome. J Cardiovasc Surg (Torino) 2010;51:305-12.

8. Smith AD, Schoenhagen P. CT imaging for acute aortic syndrome. Cleve Clin J Med 2008;75:7-9, 12, 5-7 passim.

9. Schieda N, Blaichman JI, Costa AF, Glikstein R, Hurrell C, James M, Jabehdar Maralani P, Shabana W, Tang A, Tsampalieros A, van der Pol CB, Hiremath S. Gadolinium-Based Contrast Agents in Kidney Disease: A Comprehensive Review and Clinical Practice Guideline Issued by the Canadian Association of Radiologists. Can J Kidney Health Dis 2018;5:2054358118778573.

10. Khawaja AZ, Cassidy DB, Al Shakarchi J, McGrogan DG, Inston NG, Jones RG. Revisiting the risks of MRI with Gadolinium based contrast agents-review of literature and guidelines. Insights Imaging 2015;6:553-8.

11. Karamitsos TD, Karvounis H. Magnetic resonance imaging is a safe technique in patients with prosthetic heart valves and coronary stents. Hellenic J Cardiol 2019;60:38-9.

12. Baikoussis NG, Apostolakis E, Papakonstantinou NA, Sarantitis I, Dougenis D. Safety of magnetic resonance imaging in patients with implanted cardiac prostheses and metallic cardiovascular electronic devices. Ann Thorac Surg 2011;91:2006-11.

13. Castañer E, Andreu M, Gallardo X, Mata JM, Cabezuelo MA, Pallardo Y. CT in nontraumatic acute thoracic aortic disease: typical and atypical features and complications. Radiographics 2003;23 Spec No:S93-110.

14. Thoongsuwan N, Stern EJ. Chest CT scanning for clinical suspected thoracic aortic dissection: beware the alternate diagnosis. Emerg Radiol 2002;9:257-61.

15. Yoo SM, Lee HY, White CS. MDCT evaluation of acute aortic syndrome. Radiol Clin North Am 2010;48:67-83.

16. McMahon MA, Squirrell CA. Multidetector CT of Aortic Dissection: A Pictorial Review. Radiographics 2010;30:445-60.

17. Hasebroock KM, Serkova NJ. Toxicity of MRI and 
CT contrast agents. Expert Opin Drug Metab Toxicol 2009;5:403-16.

18. Sun J, Zhang Q, Hu D, Shen Y, Yang H, Chen C, Zhou Z, Peng Y. Feasibility study of using one-tenth $\mathrm{mSv}$ radiation dose in young children chest CT with $80 \mathrm{kVp}$ and modelbased iterative reconstruction. Sci Rep 2019;9:12481.

19. Kataria B, Nilsson Althen J, Smedby O, Persson A, Sokjer $\mathrm{H}$, Sandborg M. Image quality and pathology assessment in CT Urography: when is the low-dose series sufficient? BMC Med Imaging 2019;19:64.

20. Carriero A, Magarelli N, Padovano F, Baratto M, Fossaceca R, Bonomo L. Magnetic resonance angiography of the origins of the supraaortic arteries: comparison of single and double volume acquisition 3D time of flight. J Neuroradiol 1996;23:62-8.

21. Yucel EK, Kaufman JA, Geller SC, Waltman AC. Atherosclerotic occlusive disease of the lower extremity: prospective evaluation with two-dimensional time-of-flight MR angiography. Radiology 1993;187:637-41.

22. Clough RE, Hussain T, Uribe S, Greil GF, Razavi R, Taylor PR, Schaeffter T, Waltham M. A new method for quantification of false lumen thrombosis in aortic dissection using magnetic resonance imaging and a blood pool contrast agent. J Vasc Surg 2011;54:1251-8.

23. Garcia J, Liu SZ, Louie AY. Biological effects of MRI contrast agents: gadolinium retention, potential

Cite this article as: Chen CW, Tseng YH, Lin CC, Kao CC, Wong MY, Ting H, Huang YK. Aortic dissection assessment by 4D phase-contrast MRI with hemodynamic parameters: the impact of stent type. Quant Imaging Med Surg 2021;11(2):490501. doi: 10.21037/qims-20-670 mechanisms and a role for phosphorus. Philos Trans A Math Phys Eng Sci 2017;375.

24. Layne KA, Wood DM, Dargan PI. Gadolinium-based contrast agents - what is the evidence for 'gadolinium deposition disease' and the use of chelation therapy? Clin Toxicol (Phila) 2020;58:151-60.

25. Sträter A, Huber A, Rudolph J, Berndt M, Rasper M, Rummeny EJ, Nadjiri J. 4D-Flow MRI: Technique and Applications. Rofo 2018;190:1025-35.

26. Clough RE, Waltham M, Giese D, Taylor PR, Schaeffter T. A new imaging method for assessment of aortic dissection using four-dimensional phase contrast magnetic resonance imaging. J Vasc Surg 2012;5 5:914-23.

27. Liu D, Fan Z, Li Y, Zhang N, Sun Z, An J, Stalder AF, Greiser A, Liu J. Quantitative study of abdominal blood flow patterns in patients with aortic dissection by 4-dimensional flow MRI. Sci Rep 2018;8:9111.

28. Bashir MR, Ferral H, Jacobs C, McCarthy W, Goldin M. Endoleaks after endovascular abdominal aortic aneurysm repair: management strategies according to CT findings. AJR Am J Roentgenol 2009;192:W178-86.

29. Lee YL, Huang YK, Hsu LS, Chen PY, Chen CW. The use of non-contrast-enhanced MRI to evaluate serial changes in endoleaks after aortic stenting: a case report. BMC Med Imaging 2019;19:82. 